\title{
Vector valued nonuniform nonstationary wavelets and associated MRA on local fields
}

\author{
O. AHMAD, A. H. WANI, N. A. SHEIKH AND M. AHMAD
}

\begin{abstract}
In this paper we study nonstationary wavelets associated with vector valued nonuniform multiresolution analysis on local fields. By virtue of dimension function a complete characterization of vector valued nonuniform nonstationary wavelets is obtained.
\end{abstract}

Mathematics Subject Classification 2010: 42C40; 42C15; 43A70

Keywords: Nonuniform nonstationary wavelet, Scaling function, Nonuniform nonstationary MRA, Dimension function Local field.

\section{INTRODUCTION}

In order to systematically construct orthonormal wavelet bases Mallat and Meyer introduced in 1986 the multiresolution analysis (or multiscale approximation) as a general tool in ap- proximation theory and signal analysis. Thus they provided a natural framework for the understanding of wavelet bases and provided a well structured scheme which describes the various refinement steps clearly, such that this technique became accessible to engineers for practical implementation [36]. The concept of MRA has been extended in various ways in recent years. These concepts are generalized to $L^{2}\left(\mathbb{R}^{d}\right)$, to lattices different from $\mathbb{Z}^{d}$, allowing the subspaces of MRA to be generated by Riesz basis instead of orthonormal basis, admitting a finite number of scaling functions, replacing the dilation factor 2 by an integer $M \geq 2$ or by an expansive matrix $A \in G L_{d}(\mathbb{R})$ as long as $A \subset A \mathbb{Z}^{d}$. All these concepts are developed on regular lattices, that is the translation set is always a group. Recently, Gabardo and Nashed [22] considered a generalization of Mallat's [35] celebrated theory of MRA based on spectral pairs, in which the translation set acting on the scaling function associated with the MRA to generate the subspace $V_{0}$ is no longer a group, but is the union of $\mathbb{Z}$ and a translate of $\mathbb{Z}$. Based on one-dimensional spectral pairs, Gabardo and Yu [23] considered sets of nonuniform wavelets in $L^{2}(\mathbb{R})$. In real life application all signals are not obtained from uniform shifts; so there is a natural question regarding analysis and decompositions of these types of signals by a stable 
mathematical tool. Gabardo and Nashed [22] and Gabardo and Yu [23] filled this gap by the concept of nonuniform multiresolution analysis. The notion of nonstationary wavelet system is introduced independently by M. Z. Berkolayko, I. Y. Novikov [15] and by C. de Boor, R. DeVore, A. Ron [16]. In [16], the nonstationary system (called almost-wavelets) is used to construct an orthonormal shift invariant basis consisting of infinitely differ- entiable compactly supported functions. It is well known that it is impossible to construct stationary wavelet basis satisfying these properties. Further, nonstationary wavelets are studied in $[14 ; 19]$

During the last two decades, there is a substantial body of work that has been concerned with the construction of wavelets on local fields. Even though the structures and metrics of local fields of zero and positive characteristics are similar, their wavelet and MRA (multiresolution analysis) theories are quite different. For example, R. L. Benedetto and J. J. Benedetto [13] developed a wavelet theory for local fields and related groups. They did not develop the multiresolution analysis (MRA) approach, their method is based on the theory of wavelet sets and only allows the construction of wavelet functions whose Fourier transforms are characteristic functions of some sets. Khrennikov, Shelkovich and Skopina [26] constructed a number of scaling functions generating an MRA of $L^{2}\left(\mathbb{Q}_{p}\right)$. But later on in [10], Albeverio, Evdokimov and Skopina proved that all these scaling functions lead to the same Haar MRA and that there exist no other orthogonal test scaling functions generating an MRA except those described in [26]. Some wavelet bases for $L^{2}\left(\mathbb{Q}_{p}\right)$ different from the Haar system were constructed in $[9 ; 18]$. These wavelet bases were obtained by relaxing the basic condition in the definition of an MRA and form Riesz bases without any dual wavelet systems. For some related works on wavelets and frames on $\mathbb{Q}_{p}$, we refer to $[11 ; 25 ; 29 ; 30]$. On the other hand, Lang $[31 ; 32 ; 33]$ constructed several examples of compactly supported wavelets for the Cantor dyadic group. Farkov $[20 ; 21]$ has constructed many examples of wavelets for the Vilenkin p-groups. Jiang et al. [24] pointed out a method for constructing orthogonal wavelets on local field $\mathbb{K}$ with a constant generating sequence and derived necessary and sufficient conditions for a solution of the refinement equation to generate a multiresolution analysis of $L^{2}(\mathbb{K})$.

Recently, Shah and Abdullah [39] have generalized the concept of multiresolution analysis on Euclidean spaces $\mathbb{R}^{n}$ to nonuniform multiresolution analysis on local fields of positive characteristic, in which the translation set acting 
on the scaling function associated with the multiresolution analysis to generate the subspace $V_{0}$ is no longer a group, but is the union of $\mathscr{Z}$ and a translate of $\mathscr{Z}$, where $\mathscr{Z}=\left\{u(n): n \in \mathbb{N}_{0}\right\}$ is a complete list of (distinct) coset representation of the unit disc $\mathfrak{D}$ in the locally compact Abelian group $\mathbb{K}^{+}$. The notion of nonuniform wavelet frames on non-Archimedean local fields was introduced by Ahmad and Sheikh [7] and established a complete characterization of tight nonuniform wavelet frames on non-Archimedean local fields. More results in this direction can also be found in $[1$; $2 ; 3 ; 4 ; 5 ; 6 ; 8 ; 34 ; 37 ; 38]$ and the references therein. Drawing the inspiration from the above work, we introduce the notion of nonuniform nonstationary wavelets, their characterization and the associated multiresolution analysis on local fields.

The remainder of the paper is as follows. In Section 2, we discuss preliminary results on local fields and some basic definitions. In section 3, we obtain the characterization of orthonormal vector valued nonuniform nonstationary wavelets and the associated multiresolution analysis is established.

\section{PRELIMINARIES ON NON-ARCHIMEDEAN LOCAL FIELDS}

\subsection{Non-Archimedean Local Fields}

A non-Archimedean local field $\mathbb{K}$ is a locally compact, non-discrete and totally disconnected field. If it is of characteristic zero, then it is a field of $p$-adic numbers $\mathbb{Q}_{p}$ or its finite extension. If $\mathbb{K}$ is of positive characteristic, then $\mathbb{K}$ is a field of formal Laurent series over a finite field $G F\left(p^{c}\right)$. If $c=1$, it is a $p$-series field, while for $c \neq 1$, it is an algebraic extension of degree $c$ of a $p$-series field. Let $\mathbb{K}$ be a fixed non-Archimedean local field with the ring of integers $\mathfrak{D}=\{x \in K:|x| \leq 1\}$. Since $K^{+}$is a locally compact Abelian group, we choose a Haar measure $d x$ for $K^{+}$. The field $K$ is locally compact, non-trivial, totally disconnected and complete topological field endowed with non-Archimedean norm $|\cdot|: \mathbb{K} \rightarrow \mathbb{R}^{+}$satisfying

(a) $|x|=0$ if and only if $x=0$;

(b) $|x y|=|x||y|$ for all $x, y \in \mathbb{K}$;

(c) $|x+y| \leq \max \{|x|,|y|\}$ for all $x, y \in \mathbb{K}$.

Property (c) is called the ultrametric inequality. Let $\mathfrak{B}=\{x \in \mathbb{K}:|x|<1\}$ be the prime ideal of the ring of integers $\mathfrak{D}$ in $\mathbb{K}$. Then, the residue space $\mathfrak{D} / \mathfrak{B}$ is isomorphic 
to a finite field $G F(q)$, where $q=p^{c}$ for some prime $p$ and $c \in \mathbb{N}$. Since $K$ is totally disconnected and $\mathfrak{B}$ is both prime and principal ideal, so there exist a prime element $\mathfrak{p}$ of $\mathbb{K}$ such that $\mathfrak{B}=\langle\mathfrak{p}\rangle=\mathfrak{p} \mathfrak{D}$. Let $\mathfrak{D}^{*}=\mathfrak{D} \backslash \mathfrak{B}=\{x \in \mathbb{K}:|x|=1\}$. Clearly, $\mathfrak{D}^{*}$ is a group of units in $\mathbb{K}^{*}$ and if $x \neq 0$, then can write $x=\mathfrak{p}^{n} y, y \in \mathfrak{D}^{*}$. Moreover, if $\mathscr{U}=\left\{a_{m}: m=0,1, \ldots, q-1\right\}$ denotes the fixed full set of coset representatives of $\mathfrak{B}$ in $\mathfrak{D}$, then every element $x \in K$ can be expressed uniquely as $x=\sum_{\ell=k}^{\infty} c_{\ell} \mathfrak{p}^{\ell}$ with $c_{\ell} \in \mathscr{U}$. Recall that $\mathfrak{B}$ is compact and open, so each fractional ideal $\mathfrak{B}^{k}=\mathfrak{p}^{k} \mathfrak{D}=$ $\left\{x \in K:|x|<q^{-k}\right\}$ is also compact and open and is a subgroup of $K^{+}$. We use the notation in Taibleson's book [40]. In the rest of this paper, we use the symbols $\mathbb{N}, \mathbb{N}_{0}$ and $\mathbb{Z}$ to denote the sets of natural, non-negative integers and integers, respectively.

Let $\chi$ be a fixed character on $K^{+}$that is trivial on $\mathfrak{D}$ but non-trivial on $\mathfrak{B}^{-1}$. Therefore, $\chi$ is constant on cosets of $\mathfrak{D}$ so if $y \in \mathfrak{B}^{k}$, then $\chi_{y}(x)=\chi(y, x), x \in K$. Suppose that $\chi_{u}$ is any character on $K^{+}$, then the restriction $\chi_{u} \mid \mathfrak{D}$ is a character on $\mathfrak{D}$. Moreover, as characters on $\mathfrak{D}, \chi_{u}=\chi_{v}$ if and only if $u-v \in \mathfrak{D}$. Hence, if $\left\{u(n): n \in \mathbb{N}_{0}\right\}$ is a complete list of distinct coset representative of $\mathfrak{D}$ in $K^{+}$, then, as it was proved in [40], the set $\left\{\chi_{u(n)}: n \in \mathbb{N}_{0}\right\}$ of distinct characters on $\mathfrak{D}$ is a complete orthonormal system on $\mathfrak{D}$.

We now impose a natural order on the sequence $\{u(n)\}_{n=0}^{\infty}$. We have $\mathfrak{D} / \mathfrak{B} \cong$ $G F(q)$ where $G F(q)$ is a $c$-dimensional vector space over the field $G F(p)$. We choose a set $\left\{1=\zeta_{0}, \zeta_{1}, \zeta_{2}, \ldots, \zeta_{c-1}\right\} \subset \mathfrak{D}^{*}$ such that $\operatorname{span}\left\{\zeta_{j}\right\}_{j=0}^{c-1} \cong G F(q)$. For $n \in \mathbb{N}_{0}$ satisfying

$$
0 \leq n<q, n=a_{0}+a_{1} p+\cdots+a_{c-1} p^{c-1}, 0 \leq a_{k}<p, \text { and } k=0,1, \ldots, c-1,
$$

we define

$$
u(n)=\left(a_{0}+a_{1} \zeta_{1}+\cdots+a_{c-1} \zeta_{c-1}\right) \mathfrak{p}^{-1}
$$

Also, for $n=b_{0}+b_{1} q+b_{2} q^{2}+\cdots+b_{s} q^{s}, n \in \mathbb{N}_{0}, 0 \leq b_{k}<q, k=0,1,2, \ldots, s$, we set

$$
u(n)=u\left(b_{0}\right)+u\left(b_{1}\right) \mathfrak{p}^{-1}+\cdots+u\left(b_{s}\right) \mathfrak{p}^{-s} .
$$

This defines $u(n)$ for all $n \in \mathbb{N}_{0}$. In general, it is not true that $u(m+n)=u(m)+u(n)$. But, if $r, k \in \mathbb{N}_{0}$ and $0 \leq s<q^{k}$, then $u\left(r q^{k}+s\right)=u(r) \mathfrak{p}^{-k}+u(s)$. Further, it is also easy to verify that $u(n)=0$ if and only if $n=0$ and $\left\{u(s)+u(k): k \in \mathbb{N}_{0}\right\}=\left\{u(k): k \in \mathbb{N}_{0}\right\}$ 
for a fixed $s \in \mathbb{N}_{0}$. Hereafter we use the notation $\chi_{n}=\chi_{u(n)}, n \geq 0$.

Let the local field $\mathbb{K}$ be of characteristic $p>0$ and $\zeta_{0}, \zeta_{1}, \zeta_{2}, \ldots, \zeta_{c-1}$ be as above. We define a character $\chi$ on $K$ as follows:

$$
\chi\left(\zeta_{\mu} \mathfrak{p}^{-j}\right)= \begin{cases}\exp (2 \pi i / p), & \mu=0 \text { and } j=1, \\ 1, & \mu=1, \ldots, c-1 \text { or } j \neq 1 .\end{cases}
$$

\subsection{Fourier Transforms on Non-Archimedean Local Fields}

The Fourier transform of $f \in L^{1}(\mathbb{K})$ is denoted by $\hat{f}(\xi)$ and defined by

$$
\mathscr{F}\{f(x)\}=\hat{f}(\xi)=\int_{\mathbb{K}} f(x) \overline{\chi_{\xi}(x)} d x .
$$

It is noted that

$$
\hat{f}(\xi)=\int_{\mathbb{K}} f(x) \overline{\chi_{\xi}(x)} d x=\int_{\mathbb{K}} f(x) \chi(-\xi x) d x .
$$

The properties of Fourier transforms on non-Archimedean local field $\mathbb{K}$ are much similar to those of on the classical field $\mathbb{R}$. In fact, the Fourier transform on nonArchimedean local fields of positive characteristic have the following properties:

-The map $f \rightarrow \hat{f}$ is a bounded linear transformation of $L^{1}(\mathbb{K})$ into $L^{\infty}(\mathbb{K})$, and $\|\hat{f}\|_{\infty} \leq\|f\|_{1}$.

-If $f \in L^{1}(\mathbb{K})$, then $\hat{f}$ is uniformly continuous.

-If $f \in L^{1}(\mathbb{K}) \cap L^{2}(\mathbb{K})$, then $\|\hat{f}\|_{2}=\|f\|_{2}$.

The Fourier transform of a function $f \in L^{2}(\mathbb{K})$ is defined by

$$
\hat{f}(\xi)=\lim _{k \rightarrow \infty} \hat{f}_{k}(\xi)=\lim _{k \rightarrow \infty} \int_{|x| \leq q^{k}} f(x) \overline{\chi_{\xi}(x)} d x,
$$

where $f_{k}=f \Phi_{-k}$ and $\Phi_{k}$ is the characteristic function of $\mathfrak{B}^{k}$. Furthermore, if $f \in$ $L^{2}(\mathfrak{D})$, then we define the Fourier coefficients of $f$ as

$$
\hat{f}(u(n))=\int_{\mathfrak{D}} f(x) \overline{\chi_{u(n)}(x)} d x .
$$

The series $\sum_{n \in \mathbb{N}_{0}} \hat{f}(u(n)) \chi_{u(n)}(x)$ is called the Fourier series of $f$. From the standard $L^{2}$-theory for compact Abelian groups, we conclude that the Fourier series of $f$ 
converges to $f$ in $L^{2}(\mathfrak{D})$ and Parseval's identity holds:

$$
\|f\|_{2}^{2}=\int_{\mathfrak{D}}|f(x)|^{2} d x=\sum_{n \in \mathbb{N}_{0}}|\hat{f}(u(n))|^{2} .
$$

We also denote the test function space on $K$ by $\Omega$, i.e., each function $f$ in $\Omega$ is a finite linear combination of functions of the form $\mathbf{1}_{k}(x-h), h \in K, k \in \mathbb{Z}$, where $\mathbf{1}_{k}$ is the characteristic function of $\mathfrak{B}^{k}$. Then, it is clear that $\Omega$ is dense in $L^{p}(K), 1 \leq p<\infty$, and each function in $\Omega$ is of compact support and so is its Fourier transform. Since $\Omega$ is dense in $L^{2}(K)$ and closed under the Fourier transform, the set

$$
\Omega^{0}=\{f \in \Omega: \operatorname{supp} \hat{f} \subset K \backslash\{0\}\}
$$

is also dense in $L^{2}(\mathbb{K})$.

\subsection{Uniform Stationary MRA on Local Fields}

In order to be able to define the concepts of uniform MRA and wavelets on nonArchimedean local fields, we need analogous notions of translation and dilation. Since $\bigcup_{j \in \mathbb{Z}} \mathfrak{p}^{-j} \mathfrak{D}=\mathbb{K}$, we can regard $\mathfrak{p}^{-1}$ as the dilation and since $\left\{u(n): n \in \mathbb{N}_{0}\right\}$ is a complete list of distinct coset representatives of $\mathfrak{D}$ in $K$, the set $\mathscr{Z}=\left\{u(n): n \in \mathbb{N}_{0}\right\}$ can be treated as the translation set. Note that $\Lambda$ is a subgroup of $\mathbb{K}^{+}$and unlike the standard wavelet theory on the real line, the translation set is not a group. Let us recall the definition of a uniform MRA on non-Archimedean local fields of positive characteristic introduced by Jiang et al. in [24].

DEFINITION 2.1. Let $\mathbb{K}$ be a non-Archimedean local field of positive characteristic $p>0$ and $\mathfrak{p}$ be a prime element of $\mathbb{K}$. An MRA of $L^{2}(\mathbb{K})$ is a sequence of closed subspaces $\left\{V_{j}: j \in \mathbb{Z}\right\}$ of $L^{2}(\mathbb{K})$ satisfying the following properties:

(a) $\quad V_{j} \subset V_{j+1}$ for all $j \in \mathbb{Z}$;

(b) $\bigcup_{j \in \mathbb{Z}} V_{j}$ is dense in $L^{2}(\mathbb{K})$;

(c) $\bigcap_{j \in \mathbb{Z}} V_{j}=\{0\}$;

(d) $\quad f(x) \in V_{j}$ if and only if $f\left(\mathfrak{p}^{-1} x\right) \in V_{j+1}$ for all $j \in \mathbb{Z}$;

(e) There exists a function $\phi \in V_{0}$, such that $\left\{\phi(x-u(k)): k \in \mathbb{N}_{0}\right\}$ forms an orthonormal basis for $V_{0}$. 
According to the standard scheme for construction of MRA-based wavelets, for each $j$, we define a wavelet space $W_{j}$ as the orthogonal complement of $V_{j}$ in $V_{j+1}$, i.e., $V_{j+1}=V_{j} \oplus W_{j}, j \in \mathbb{Z}$, where $W_{j} \perp V_{j}, j \in \mathbb{Z}$. It is not difficult to see that

$$
f(x) \in W_{j} \quad \text { if and only if } \quad f\left(\mathfrak{p}^{-1} x\right) \in W_{j+1}, \quad j \in \mathbb{Z} .
$$

Moreover, they are mutually orthogonal, and we have the following orthogonal decompositions:

$$
L^{2}(\mathbb{K})=\bigoplus_{j \in \mathbb{Z}} W_{j}=V_{0} \oplus\left(\bigoplus_{j \geq 0} W_{j}\right) .
$$

As in the case of $\mathbb{R}^{n}$, we expect the existence of $q-1$ number of functions $\psi_{1}, \psi_{2}, \ldots, \psi_{q-1}$ to form a set of basic wavelets. In view of (2.7) and (2.8), it is clear that if $\left\{\psi_{1}, \psi_{2}, \ldots, \psi_{q-1}\right\}$ is a set of function such that the system $\left\{\psi_{\ell}(x-u(k)): 1 \leq \ell \leq q-1, k \in \mathbb{N}_{0}\right\}$ forms an orthonormal basis for $W_{0}$, then $\left\{q^{j / 2} \psi_{\ell}\left(\mathfrak{p}^{-j} x-u(k)\right): 1 \leq \ell \leq q-1, j \in \mathbb{Z}, k \in \mathbb{N}_{0}\right\}$ forms an orthonormal basis for $L^{2}(\mathbb{K})$.

\subsection{Nonuniform MRA on Non-Archimedean Local Fields}

For an integer $N \geq 1$ and an odd integer $r$ with $1 \leq r \leq q N-1$ such that $r$ and $N$ are relatively prime, we define

$$
\Lambda=\left\{0, \frac{u(r)}{N}\right\}+\mathscr{Z} .
$$

where $\mathscr{Z}=\left\{u(n): n \in \mathbb{N}_{0}\right\} . \quad$ It is easy to verify that $\Lambda$ is not a group on non-Archimedean local field $\mathbb{K}$, but is the union of $\mathscr{Z}$ and a translate of $\mathscr{Z}$. Following is the definition of nonuniform stationary multiresolution analysis (NUSMRA) on non-Archimedean local fields of positive characteristic given by Shah and Abdullah [39].

DEFINITION 2.2. For an integer $N \geq 1$ and an odd integer $r$ with $1 \leq r \leq q N-1$ such that $r$ and $N$ are relatively prime, an associated NUMRA on non-Archimedean local field $\mathbb{K}$ of positive characteristic is a sequence of closed subspaces $\left\{V_{j}: j \in \mathbb{Z}\right\}$ of $L^{2}(\mathbb{K})$ such that the following properties hold:

(a) $\quad V_{j} \subset V_{j+1}$ for all $j \in \mathbb{Z}$; 
(b) $\bigcup_{j \in \mathbb{Z}} V_{j}$ is dense in $L^{2}(\mathbb{K})$;

(c) $\bigcap_{j \in \mathbb{Z}} V_{j}=\{0\}$;

(d) $f(\cdot) \in V_{j}$ if and only if $f\left(\mathfrak{p}^{-1} N \cdot\right) \in V_{j+1}$ for all $j \in \mathbb{Z}$;

(e) There exists a function $\phi$ in $V_{0}$ such that $\{\phi(\cdot-\lambda): \lambda \in \Lambda\}$, is a complete orthonormal basis for $V_{0}$.

It is worth noticing that, when $N=1$, one recovers from the definition above the definition of an MRA on non-Archimedean local fields of positive characteristic $p>0$. When, $N>1$, the dilation is induced by $\mathfrak{p}^{-1} N$ and $\left|\mathfrak{p}^{-1}\right|=q$ ensures that $q N \Lambda \subset \mathscr{Z} \subset \Lambda$.

For every $j \in \mathbb{Z}$, define $W_{j}$ to be the orthogonal complement of $V_{j}$ in $V_{j+1}$. Then we have

$$
V_{j+1}=V_{j} \oplus W_{j} \quad \text { and } \quad W_{\ell} \perp W_{\ell^{\prime}} \quad \text { if } \ell \neq \ell^{\prime} .
$$

It follows that for $j>J$,

$$
V_{j}=V_{J} \oplus \bigoplus_{\ell=0}^{j-J-1} W_{j-\ell},
$$

where all these subspaces are orthogonal. By virtue of condition (b) in the Definition 2.2 , this implies

$$
L^{2}(\mathbb{K})=\bigoplus_{j \in \mathbb{Z}} W_{j}
$$

a decomposition of $L^{2}(\mathbb{K})$ into mutually orthogonal subspaces.

Now we state the concept of vector-valued nonuniform multiresolution analysis (VNUMRA) on local field $K$ of positive characteristic and establish a necessary and sufficient condition for the existence of associated wavelets.

Let $M$ be a constant and $2 \leq M \in \mathbb{Z}$. By $L^{2}\left(K, \mathbb{C}^{M}\right)$, we denote the set of all vectorvalued functions $\mathbf{f}$ i.e.,

$$
L^{2}\left(K, \mathbb{C}^{M}\right)=\left\{\mathbf{f}(x)=\left(f_{1}(x), f_{2}(x), \ldots, f_{M}(x)\right)^{T}: x \in K, f_{t}(x) \in L^{2}(K)\right\},
$$

where $t=1,2, \ldots, M$ and $T$ means the transpose of a vector. The space $L^{2}\left(K, \mathbb{C}^{M}\right)$ is called vector-valued function space on local field $K$ of positive characteristic. For 
$\mathbf{f} \in L^{2}\left(K, \mathbb{C}^{M}\right),\|\mathbf{f}\|$ denotes the norm of vector-valued function $\mathbf{f}$ and is defined as:

$$
\|\mathbf{f}\|_{2}=\left(\sum_{t=1}^{M} \int_{K}\left|f_{t}(x)\right|^{2} d x\right)^{1 / 2} .
$$

For a vector-valued function $\mathbf{f} \in L^{2}\left(K, \mathbb{C}^{M}\right)$, the integration of $\mathbf{f}$ is defined as:

$$
\int_{K} \mathbf{f}(x) d x=\left(\int_{K} f_{1}(x) d x, \int_{K} f_{2}(x) d x, \ldots, \int_{K} f_{M}(x) d x\right)^{T} .
$$

Moreover, the Fourier transform of $\mathbf{f}$ is defined by

$$
\hat{\mathbf{f}}(\xi)=\int_{K} \mathbf{f}(x) \overline{\chi_{\xi}(x)} d x .
$$

For any two vector-valued functions $\mathbf{f}, \mathbf{g} \in L^{2}\left(K, \mathbb{C}^{M}\right)$, their vector-valued inner product $\langle\mathbf{f}, \mathbf{g}\rangle$ is defined as:

$$
\langle\mathbf{f}, \mathbf{g}\rangle=\int_{K} \mathbf{f}(x) \overline{\mathbf{g}(x)} d x .
$$

With $\Lambda=\{0, r / N\}+\mathscr{Z}$ as defined above, we define the vector-valued nonuniform multiresolution analysis (VNUMRA) on local fields of positive characteristic as follows:

DEFINITION 2.3. For an integer $N \geq 1$ and an odd integer $r$ with $1 \leq r \leq$ $q N-1$ such that $r$ and $N$ are relatively prime, a VNUMRA on local field $K$ of positive characteristic is a sequence of closed subspaces $\left\{V_{j}: j \in \mathbb{Z}\right\}$ of $L^{2}\left(K, \mathbb{C}^{M}\right)$ such that the following properties hold:

(1) $V_{j} \subset V_{j+1}$ for all $j \in \mathbb{Z}$;

(2) $\bigcup_{j \in \mathbb{Z}} V_{j}$ is dense in $L^{2}\left(K, \mathbb{C}^{M}\right)$;

(3) $\bigcap_{j \in \mathbb{Z}} V_{j}=\{\mathbf{0}\}$, where $\mathbf{0}$ is the zero vector of $L^{2}\left(K, \mathbb{C}^{M}\right)$;

(4) $\Phi(x) \in V_{j}$ if and only if $\Phi\left(\mathfrak{p}^{-1} N x\right) \in V_{j+1}$ for all $j \in \mathbb{Z}$;

(5) There exists a function $\Phi$ in $V_{0}$ such that $\{\Phi(x-\lambda): \lambda \in \Lambda\}$, is a complete orthonormal basis for $V_{0}$. The vector valued function $\Phi$ is called a vector-valued scaling function of the VNUMRA. 
For every $j \in \mathbb{Z}$, define $W_{j}$ to be the orthogonal complement of $V_{j}$ in $V_{j+1}$. Then we have

$$
V_{j+1}=V_{j} \oplus W_{j} \quad \text { and } \quad W_{\ell} \perp W_{\ell^{\prime}} \quad \text { if } \ell \neq \ell^{\prime} .
$$

It follows that for $j>J$,

$$
V_{j}=V_{J} \bigoplus\left(\bigoplus_{\ell=0}^{j-J-1} W_{j-\ell}\right)
$$

where all these subspaces are orthogonal. By virtue of condition (b) in the Definition 2.3 , this implies

$$
L^{2}\left(K, \mathbb{C}^{M}\right)=\bigoplus_{j \in \mathbb{Z}} W_{j},
$$

a decomposition of $L^{2}\left(K, \mathbb{C}^{M}\right)$ into mutually orthogonal subspaces.

As in the standard case, one expects the existence of $q N-1$ number of functions so that their translation by elements of $\Lambda$ and dilations by the integral powers of $\mathfrak{p}^{-1} N$ form an orthonormal basis for $L^{2}\left(K, \mathbb{C}^{M}\right)$.

DEFINITION 2.4. A set of functions $\left\{\Psi_{1}, \Psi_{2}, \ldots, \Psi_{q N-1}\right\}$ in $L^{2}\left(K, \mathbb{C}^{M}\right)$ will be called a set of basic wavelets associated with a given VNUMRA if the family of functions $\left\{\Psi_{\ell}(x-\lambda): 1 \leq \ell \leq q N-1, \lambda \in \Lambda\right\}$ forms an orthonormal basis for $W_{0}$.

\section{MAIN RESULTS}

We start this section with the following definition

DEFINITION 3.1. Let $\Psi^{(j)} \in L^{2}\left(K, \mathbb{C}^{M}\right)$ for all $j \in \mathbb{Z}$, then the family of functions

$$
\Psi_{j, \lambda}=\left\{(q N)^{\frac{j}{2}} \Psi^{(j)}\left(\mathfrak{p}^{-1} N\right)^{j} x-\lambda\right\}_{j \in \mathbb{Z}, \lambda \in \Lambda}
$$

is called a nonuniform nonstationary wavelet system for $L^{2}\left(K, \mathbb{C}^{M}\right)$.

LEMMA 3.1. If $f \in S$ and $\Psi^{(j)} \in L^{2}\left(K, \mathbb{C}^{M}\right)$, then

$$
\sum_{\lambda \in \Lambda}\left|\left\langle f, \Psi_{j, \lambda}\right\rangle\right|^{2}=\int_{\mathbb{K}} \overline{\widehat{f}}(\xi) \widehat{\Psi}^{(j)}\left(\left(\mathfrak{p}^{-1} N\right)^{-j} \xi\right)\left\{\sum_{s \in \mathbb{N}_{0}} \widehat{f}\left(\xi+\left(\mathfrak{p}^{-1} N\right)^{-j} u(s)\right) \overline{\widehat{\Psi}^{(j)}}\left(\left(\mathfrak{p}^{-1} N\right)^{-j} \xi+u(s)\right)\right\} d \xi .
$$


PROOF. For $\Psi \in L^{2}\left(K, \mathbb{C}^{M}\right)$, let

$$
\Psi_{j, \lambda}(x)=(q N)^{j / 2} \Psi^{(j)}\left(\left(\mathfrak{p}^{-1} N\right)^{j} x-\lambda\right), j \in \mathbb{Z}, \lambda \in \Lambda
$$

Then, we have

$$
\widehat{\Psi}_{j, \lambda}(\xi)=(q N)^{-j / 2} \widehat{\Psi}^{(j)}\left(\left(\mathfrak{p}^{-1} N\right)^{-j} \xi\right) \overline{\chi_{\lambda}\left(\left(\mathfrak{p}^{-1} N\right)^{-j} \xi\right)} .
$$

By Parseval Identity and equation (3.2), we have

$$
\begin{aligned}
& \sum_{\lambda \in \Lambda}\left|\left\langle f, \Psi_{j, \lambda}\right\rangle\right|^{2}=\sum_{\lambda \in \Lambda}(q N)^{j} \int_{\mathbb{K}}\left\{\sum_{s \in \mathbb{N}_{0}} \int_{N \mathfrak{D}} \widehat{f}\left(\left(\mathfrak{p}^{-1} N\right)^{-j}(x+u(s))\right) \chi_{\lambda}(x+u(s)) \overline{\widehat{\Psi}^{(j)}}(x+u(s))\right\} \\
& \times \overline{\widehat{f}\left(\left(\mathfrak{p}^{-1} N\right)^{-j} x\right)} \overline{\chi_{\lambda}(x)} \widehat{\Psi}^{(j)}(x) d x .
\end{aligned}
$$

Since $\sum_{s \in \mathbb{N}_{0}} \int_{N \mathfrak{D}} \widehat{f}\left(\left(\mathfrak{p}^{-1} N\right)^{-j}(x+u(s))\right) \chi_{\lambda}(x+u(s)) \overline{\widehat{\Psi}^{(j)}}(x+u(s)) d x$ contains only finite non-zero terms for $f \in S$ and $\chi_{\lambda}(u(s))=1$, for all $\lambda \in \Lambda, s \in \mathbb{N}_{0}$, we have

$$
\begin{array}{r}
\sum_{\lambda \in \Lambda}\left|\left\langle f, \Psi_{j, \lambda}\right\rangle\right|^{2}=\sum_{\lambda \in \Lambda}(q N)^{j} \int_{\mathbb{K}}\left(\int_{N \mathfrak{D}}\left\{\sum_{s \in \mathbb{N}_{0}} \widehat{f}\left(\left(\mathfrak{p}^{-1} N\right)^{-j}(x+u(s))\right) \overline{\widehat{\Psi}^{(j)}}(x+u(s))\right\} \chi_{\lambda}(x) d x\right) \\
\times \overline{\chi_{\lambda}(y)} \overline{\widehat{f}\left(\left(\mathfrak{p}^{-1} N\right)^{-j} y\right)} \widehat{\Psi}^{(j)}(y) d y .
\end{array}
$$

By invoking Convergence theorem of Fourier series on $\mathfrak{D}$, we obtain (3.1). This completes the proof.

LEMMA 3.2. Let $f \in \Omega$ and $\Psi^{(j)} \in L^{2}\left(K, \mathbb{C}^{M}\right) . \quad$ If ess $\sup \left\{\sum_{j \in \mathbb{Z}}\left|\Psi^{(j)}\left(\left(\mathfrak{p}^{-1} N\right)^{j} \xi\right)\right|^{2}\right\}<\infty$ for $\xi \in \mathfrak{B}^{-1} \backslash N \mathfrak{D}$, then

$$
\sum_{j \in \mathbb{Z}} \sum_{\lambda \in \Lambda}\left|\left\langle f, \Psi_{j, \lambda}\right\rangle\right|^{2}=\int_{\mathbb{K}}|\widehat{f}(\xi)|^{2} \sum_{j \in \mathbb{Z}}\left|\Psi^{(j)}\left(\left(\mathfrak{p}^{-1} N\right)^{j} \xi\right)\right|^{2}+R_{0}(f)
$$


where

$$
\begin{aligned}
R_{0}(f) & =\sum_{j \in \mathbb{Z}}(q N)^{j} \int_{\mathbb{K}} \overline{\widehat{f}\left(\left(\mathfrak{p}^{-1} N\right)^{-j} \xi\right)} \Psi^{(j)}(\xi)\left\{\sum_{s \in \mathbb{N}} \widehat{f}\left(\left(\mathfrak{p}^{-1} N\right)^{-j}(\xi+u(s))\right) \overline{\widehat{\Psi}^{(j)}(\xi+u(s))}\right\} d \xi \\
& =\sum_{j \in \mathbb{Z}} \sum_{s \in \mathbb{N}}(q N)^{j} \int_{\mathbb{K}} \overline{\widehat{f}\left(\left(\mathfrak{p}^{-1} N\right)^{-j} \xi\right)} \Psi^{(j)}(\xi) \widehat{f}\left(\left(\mathfrak{p}^{-1} N\right)^{-j}(\xi+u(s))\right) \overline{\widehat{\Psi}^{(j)}(\xi+u(s))} d \xi .(3.4)
\end{aligned}
$$

Moreover if $\left\|\widehat{\Psi}^{(j)}\right\|_{L^{2}(\mathbb{K})}=1$, then the series in (3.4) converges absolutely on $\mathbb{K}$.

PROOF. For $R_{0}(f)$, we use the fact that for $f \in \Omega$, $\sum_{s \in \mathbb{N}_{0}} \widehat{f}\left(\left(\mathfrak{p}^{-1} N\right)^{-j}(x+u(s))\right) \widehat{\Psi}^{(j)}(x+u(s))$ contains only finite non-zero terms, so we have

$$
\begin{aligned}
& \sum_{j \in \mathbb{Z}}(q N)^{j} \int_{\mathbb{K}} \overline{\widehat{f}\left(\left(\mathfrak{p}^{-1} N\right)^{-j} \xi\right)} \Psi^{(j)}(\xi)\left\{\sum_{s \in \mathbb{N}} \widehat{f}\left(\left(\mathfrak{p}^{-1} N\right)^{-j}(x+u(s))\right) \overline{\widehat{\Psi}^{(j)}(x+u(s))}\right\} d \xi \\
& \quad=\sum_{j \in \mathbb{Z}} \sum_{s \in \mathbb{N}}(q N)^{j} \int_{\mathbb{K}} \overline{\widehat{f}\left(\left(\mathfrak{p}^{-1} N\right)^{-j} \xi\right)} \Psi^{(j)}(\xi) \widehat{f}\left(\left(\mathfrak{p}^{-1} N\right)^{-j}(x+u(s))\right) \overline{\widehat{\Psi}^{(j)}(x+u(s))} d \xi
\end{aligned}
$$

We claim that for all $f \in \Omega^{0}$, (3.3) holds. Moreover, by using Lemma 3.1, we have

$$
\begin{aligned}
\sum_{j \in \mathbb{Z}} \sum_{\lambda \in \Lambda}\left|\left\langle f, \Psi_{j, \lambda}\right\rangle\right|^{2} & =\sum_{j \in \mathbb{Z}} \int_{\mathbb{K}}\left\{|\widehat{f}(\xi)|^{2}\left|\widehat{\Psi}^{(j)}\left(\left(\mathfrak{p}^{-1} N\right)^{-j} \xi\right)\right|^{2}\right. \\
& \left.+(q N)^{j}\left(\overline{\widehat{f}\left(\left(\mathfrak{p}^{-1} N\right)^{-j} \xi\right)} \Psi^{(j)}(\xi) \sum_{s \in \mathbb{N}} \widehat{f}\left(\left(\mathfrak{p}^{-1} N\right)^{-j}(x+u(s))\right) \overline{\widehat{\Psi}^{(j)}(x+u(s))}\right)\right\} d \xi \\
& =\sum_{j \in \mathbb{Z}} \int_{\mathbb{K}}|\widehat{f}(\xi)|^{2}\left|\widehat{\Psi}^{(j)}\left(\left(\mathfrak{p}^{-1} N\right)^{-j} \xi\right)\right|^{2}+R_{0}(f) .
\end{aligned}
$$

Hence, our claim is true for $f \in \Omega^{0}$. Further by applying Levi Lemma and the given assumption, we obtain (3.3). We now show that the series (3.3) is absolutely convergent. Since

$$
\left|\widehat{\Psi}^{(j)}(x) \widehat{\Psi}^{(j)}(x+u(s))\right| \leq \frac{1}{2}\left(\left|\widehat{\Psi}^{(j)}(x)\right|^{2}+\left|\widehat{\Psi}^{(j)}(x+u(s))\right|^{2}\right),
$$

it suffices to verify that the series

$$
\sum_{j \in \mathbb{Z}} \sum_{s \in \mathbb{N}}(q N)^{-j} \int_{\mathbb{K}}\left|\widehat{f}\left(\left(\mathfrak{p}^{-1} N\right)^{-j} x\right) \widehat{f}\left(\left(\mathfrak{p}^{-1} N\right)^{-j}(x+u(s))\right)\right|\left|\widehat{\Psi}^{(j)}(x)\right|^{2} d x
$$


is convergent. As $u(s) \neq 0$ for $s \in \mathbb{N}$ and $f \in \Omega^{0}$, there exists a constant $J>0$ such that

$$
\widehat{f}\left(\left(\mathfrak{p}^{-1} N\right)^{-j} x\right) \widehat{f}\left(\left(\mathfrak{p}^{-1} N\right)^{-j}(x+u(s))\right)=0 \quad \forall|j|>J .
$$

On the other hand, for each fixed $|j| \leq J$, there is a constant $L$ such that

$$
\widehat{f}\left(\left(\mathfrak{p}^{-1} N\right)^{-j}(x+u(s))\right)=0 \quad \forall s>L .
$$

Hence, it means that there are only finite non-zero terms in the series (3.5). Thus, there exists a constant $C$ such that

$$
\sum_{j \in \mathbb{Z} s \in \mathbb{N}} \sum_{s}(q N)^{-j} \int_{\mathbb{K}}\left|\widehat{f}\left(\left(\mathfrak{p}^{-1} N\right)^{-j} x\right) \widehat{f}\left(\left(\mathfrak{p}^{-1} N\right)^{-j}(x+u(s))\right) \widehat{\Psi}^{(j)}(x) \widehat{\Psi}^{(j)}(x+u(s))\right| d x \leq C\|\widehat{f}\|_{\infty}^{2}\left\|\widehat{\Psi}^{(j)}\right\|_{2}^{2}
$$

Thus, it follows that the series $\sum_{j \in \mathbb{Z}} \sum_{s \in \mathbb{N}}\left|\left\langle f, \Psi_{j, \lambda}\right\rangle\right|^{2}$ is also convergent.

For given $s \in \mathbb{N}$, there is a unique pair $(\lambda, m)$ with $\lambda \in \Lambda$ and $m \in q \Lambda+\mathscr{Q}$, where $q \Lambda=\{q \lambda: \lambda \in \Lambda\}$ and $\mathscr{Q}=\{1,2, \ldots, q N-1\}$, such that $s=(q N)^{\lambda} m$. Therefore, we have $\{u(s)\}_{s \in \mathbb{N}}=\left\{\left(\mathfrak{p}^{-1} N\right)^{-\lambda} u(m)\right\}_{(\lambda, m) \in \Lambda \times(q \Lambda+\mathscr{Q})}$. Since the series (3.4) is absolutely convergent, we can estimate $R_{0}(f)$ by rearranging the series, changing the order of summation and integration by Levi Lemma as follows 


$$
\begin{aligned}
& R_{0}(f)=\sum_{j \in \mathbb{Z}}(q N)^{j} \int_{\mathbb{K}} \overline{\widehat{f}\left(\left(\mathfrak{p}^{-1} N\right)^{-j} \xi\right)} \Psi^{(j)}(\xi)\left\{\sum_{s \in \mathbb{N}} \widehat{f}\left(\left(\mathfrak{p}^{-1} N\right)^{-j}(\xi+u(s))\right) \overline{\widehat{\Psi}^{(j)}(\xi+u(s))}\right\} d \xi \\
& =\sum_{j \in \mathbb{Z}}(q N)^{j} \int_{\mathbb{K}} \overline{\widehat{f}\left(\left(\mathfrak{p}^{-1} N\right)^{-j} \xi\right)}\left\{\sum_{\lambda \in \Lambda} \sum_{m \in q \Lambda+\mathscr{Q}} \Psi^{(j)}(\xi) \widehat{f}\left(\left(\mathfrak{p}^{-1} N\right)^{-j}\left(\xi+\left(\mathfrak{p}^{-1} N\right)^{-k} u(m)\right)\right)\right. \\
& \left.\times \overline{\widehat{\Psi}^{(j)}\left(\xi+\left(\mathfrak{p}^{-1} N\right)^{-k} u(m)\right)}\right\} d \xi \\
& =\int_{\mathbb{K}} \sum_{j \in \mathbb{Z}}(q N)^{j} \overline{\widehat{f}\left(\left(\mathfrak{p}^{-1} N\right)^{-j} \xi\right)}\left\{\sum_{\lambda \in \Lambda m \in q \Lambda+\mathscr{Q}} \sum \Psi^{(j)}\left(\left(\mathfrak{p}^{-1} N\right)^{-k} \xi\right) \widehat{f}\left(\left(\mathfrak{p}^{-1} N\right)^{-j}\left(\xi+\left(\mathfrak{p}^{-1} N\right)^{-k} u(m)\right)\right)\right. \\
& \left.\times \overline{\widehat{\Psi}^{(j)}\left(\mathfrak{p}^{-1} N\right)^{-k}((\xi+u(m)))}\right\} d \xi \\
& =\int_{\mathbb{K}} \sum_{j \in \mathbb{Z}}(q N)^{j} \overline{\widehat{f}\left(\left(\mathfrak{p}^{-1} N\right)^{-j} \xi\right)}\left\{\sum_{m \in q \mathbb{N}_{0}+\mathscr{Q}} \widehat{f}\left(\left(\mathfrak{p}^{-1} N\right)^{-j}\left(\xi+\left(\mathfrak{p}^{-1} N\right)^{-k} u(m)\right)\right)\right. \\
& \left.\times \sum_{\lambda \in \Lambda} \Psi^{(j)}\left(\left(\mathfrak{p}^{-1} N\right)^{-k} \xi\right) \overline{\widehat{\Psi}^{(j)}\left(\mathfrak{p}^{-1} N\right)^{-k}((\xi+u(m)))}\right\} d \xi \\
& =\int_{\mathbb{K}} \sum_{j \in \mathbb{Z}}(q N)^{j} \overline{\widehat{f}\left(\left(\mathfrak{p}^{-1} N\right)^{-j} \xi\right)}\left\{\sum_{m \in q \mathbb{N}_{0}+\mathscr{Q}} \widehat{f}\left(\left(\mathfrak{p}^{-1} N\right)^{-j}\left(\xi+\left(\mathfrak{p}^{-1} N\right)^{-k} u(m)\right)\right) t_{\Psi(j)}(u(m), \xi)\right\} d \xi \\
& =\sum_{j \in \mathbb{Z}} \sum_{m \in q \mathbb{N}_{0}+\mathscr{Q}}(q N)^{j} \int_{\mathbb{K}} \overline{\widehat{f}} \overline{\left(\left(\mathfrak{p}^{-1} N\right)^{-j} \xi\right)} \widehat{f}\left(\left(\mathfrak{p}^{-1} N\right)^{-j}\left(\xi+\left(\mathfrak{p}^{-1} N\right)^{-k} u(m)\right)\right) t_{\Psi(j)}(u(m), \xi) d \xi,
\end{aligned}
$$

where

$$
t_{\psi^{(j)}}(u(m), \xi)=\sum_{k \in \mathbb{N}_{0}} \Psi^{(j)}\left(\left(\mathfrak{p}^{-1} N\right)^{-k} \xi\right) \overline{\widehat{\Psi}^{(j)}\left(\mathfrak{p}^{-1} N\right)^{-k}((\xi+u(m)))}
$$
Then

THEOREM 3.3. Assume that $\Psi^{(j)} \in L^{2}\left(K, \mathbb{C}^{M}\right)$ and $\left\|\widehat{\Psi}^{(j)}\right\|_{L^{2}(\mathbb{K})}=1$ for $j \in \mathbb{Z}$.

$$
\sum_{j \in \mathbb{Z}}\left|\widehat{\Psi}^{(-j)}\left(\left(\mathfrak{p}^{-1} N\right)^{-j} \xi\right)\right|^{2}=1 \quad \text { a.e } \xi \in K
$$


and

$\sum_{j=0}^{\infty} \widehat{\Psi}^{(n-j)}\left(\left(\mathfrak{p}^{-1} N\right)^{-j} \xi\right) \overline{\widehat{\Psi}^{(n-j)}\left(\left(\mathfrak{p}^{-1} N\right)^{-j}(\xi+u(m))\right)}=0 \quad$ a.e $n \in \mathbb{N}_{0}$ and $m \in q \Lambda+\mathscr{Q}$

if and only if

$$
\left\{(q N)^{j / 2} \Psi^{(j)}\left(\left(\mathfrak{p}^{-1} N\right)^{-j} x-\lambda\right), j \in \mathbb{Z}, \lambda \in \Lambda\right\}
$$

is an orthonormal basis of $L^{2}(\mathbb{K})$.

PROOF. Sufficiency part: As $\left\|\widehat{\Psi}^{(j)}\right\|_{L^{2}(\mathbb{K})}=1$, it is clear that the system $\left\{\Psi_{j, \lambda}\right.$ : $j \in \mathbb{Z}, \lambda \in \Lambda\}$ is an orthonormal basis if and only if for any $f \in L^{2}\left(K, \mathbb{C}^{M}\right)$, the Parseval identity holds.

Assume that the conditions (3.6) and (3.7) hold. Then for $n \in \mathbb{N}_{0}, m \in q \Lambda+\mathscr{Q}$, we have by Lemma 3.1 and Lemma 3.2, we have

$$
\begin{aligned}
\sum_{j \in \mathbb{Z}} \sum_{\lambda \in \Lambda}\left|\left\langle f, \Psi_{j, \lambda}\right\rangle\right|^{2} & =\int_{\mathbb{K}}|\widehat{f}(\xi)|^{2} \sum_{j \in \mathbb{Z}}\left|\widehat{\Psi}^{(j)}\left(\left(\mathfrak{p}^{-1} N\right)^{-j} \xi\right)\right|^{2} d \xi \\
& =\|f\|_{L^{2}\left(K, \mathbb{C}^{M}\right)}^{2} \quad \forall f \in S .
\end{aligned}
$$

Necessary condition: We assume that $\left\{(q N)^{j / 2} \Psi^{(j)}\left(\left(\mathfrak{p}^{-1} N\right)^{j} x-\lambda\right), j \in \mathbb{Z}, \lambda \in \Lambda\right\}$ is an orthonormal basis of $L^{2}\left(K, \mathbb{C}^{M}\right)$ and will prove the conditions (3.6) and (3.7). We assume $\Delta_{j}$ to be the set of regular points of $\left|\widehat{\Psi}^{(j)}\left(\left(\mathfrak{p}^{-1} N\right)^{-j} \xi\right)\right|^{2}$, so that for each $x \in \Delta_{j}$,

$$
(q N)^{n} \int_{\xi-x \in \mathfrak{B}^{n}}\left|\widehat{\Psi}^{(j)}\left(\left(\mathfrak{p}^{-1} N\right)^{-j} \xi\right)\right|^{2} d \xi \rightarrow\left|\widehat{\Psi}^{(j)}\left(\left(\mathfrak{p}^{-1} N\right)^{-j} x\right)\right|^{2}, \quad \text { as } n \rightarrow \infty
$$

Then $\left|\Delta_{j}^{c}\right|=0$, so that $\left|\bigcup_{j \in \mathbb{Z}} \Delta_{j}^{c}\right|=0$. Let $\xi_{0} \in \mathbb{K} \backslash \bigcup_{j \in \mathbb{Z}} \Delta_{j}^{c}$. For each fixed positive integer $M$, set

$$
\widehat{f}(\xi)=(q N)^{m / 2} \Phi_{m}\left(\xi-\xi_{0}\right), \quad m \geq M,
$$


where $\Phi_{m}\left(\xi-\xi_{0}\right)$ is the characteristic function of $\xi_{0}+\mathfrak{B}^{m}$. Then it follows that for $s \in \mathbb{N}$ and $j \geq-M, \widehat{\hat{f}(\xi)} \widehat{f}\left(\xi+\left(\mathfrak{p}^{-1} N\right)^{-j} u(s)\right)=0$, and hence $\|f\|_{2}^{2}=1$. Furthermore, we have

$$
\sum_{j \in \mathbb{Z}} \sum_{\lambda \in \Lambda}\left|\left\langle f, \Psi_{j, \lambda}\right\rangle\right|^{2}=\sum_{j \geq-M} \int_{\xi_{0}+\mathfrak{B}^{m}}(q N)^{m}|\widehat{f}(\xi)|^{2}\left|\widehat{\Psi}^{(j)}\left(\left(\mathfrak{p}^{-1} N\right)^{-j} \xi\right)\right|^{2} d \xi \leq B
$$

Therefore, in the limiting case, we have

$$
\sum_{j \in \mathbb{Z}}\left|\widehat{\Psi}^{(-j)}\left(\left(\mathfrak{p}^{-1} N\right)^{-j} \xi_{0}\right)\right|^{2}=1 \quad \text { a.e }
$$

To prove (3.7), we let

$$
\sum_{j \in \mathbb{Z}} \sum_{\lambda \in \Lambda}\left|\left\langle f, \Psi_{j, \lambda}\right\rangle\right|^{2}=I_{1}+I_{2}
$$

where

$$
I_{1}=\sum_{j>-M} \sum_{\lambda \in \Lambda}\left|\left\langle f, \Psi_{j, \lambda}\right\rangle\right|^{2} \quad \text { and } \quad I_{2}=\sum_{j \leq-M} \sum_{\lambda \in \Lambda}\left|\left\langle f, \Psi_{j, \lambda}\right\rangle\right|^{2}
$$

Since, it has been already verified that $I_{1}=\sum_{j>-M}\left|\widehat{\Psi}^{(-j)}\left(\left(\mathfrak{p}^{-1} N\right)^{-j} \xi_{0}\right)\right|^{2}$, so to prove the result, it is enough to show that $\lim _{M \rightarrow+\infty} I_{2}=0$.

Using Lemma 3.1 and Schwartz Inequality, we have

$$
\begin{aligned}
0 \leq I_{2} \leq \sum_{j \leq-M} \sum_{r \in \mathbb{N}_{0}}\left\{\int_{\mathbb{K}}\left|\widehat{\hat{f}(\xi)} \widehat{\Psi}^{(-j)}\left(\left(\mathfrak{p}^{-1} N\right)^{-j} \xi\right)\right|^{2} d \xi\right\}^{\frac{1}{2}} \\
\times\left\{\int_{\mathbb{K}}\left|\widehat{f}\left(\xi+\left(\mathfrak{p}^{-1} N\right)^{-j} u(r)\right) \overline{\widehat{\Psi}^{(-j)}\left(\left(\mathfrak{p}^{-1} N\right)^{-j} \xi+u(r)\right)}\right|^{2} d \xi\right\}^{\frac{1}{2}} .
\end{aligned}
$$

If $\xi+\left(\mathfrak{p}^{-1} N\right)^{-j} u(r) \in \xi_{0}+\mathfrak{B}^{m}$ for a fixed $j \leq-M$, then it follows that $\left|\left(\mathfrak{p}^{-1} N\right)^{-j} u(r)\right| \leq(q N)^{-m}$, so $|u(r)| \leq(q N)^{-m-j}$. Therefore

$$
I_{2} \leq \sum_{j \leq-M} \int_{\mathbb{K}}\left|\widehat{\widehat{f}(\xi)} \widehat{\Psi}^{(-j)}\left(\left(\mathfrak{p}^{-1} N\right)^{-j} \xi\right)\right|^{2} d \xi \leq \sum_{j \leq-M} \int_{\left(\mathfrak{p}^{-1} N\right)^{-j} \xi_{0}+\mathfrak{B}^{-j+m}}\left|\widehat{\Psi}^{(-j)}(\xi)\right|^{2} d \xi .
$$

If $\xi_{0} \neq 0$, then for given $\varepsilon>0$, we choose $M$ so that

$$
(q N)^{-M}<\left|\xi_{0}\right|=(q N)^{s} \quad \text { and } \quad \int_{\mathfrak{B}^{M-s}}\left|\widehat{\Psi}^{(-j)}(\xi)\right|^{2} d \xi<\varepsilon
$$


Therefore for all $j \leq-M$, we have

$$
\left(\mathfrak{p}^{-1} N\right)^{-j} \xi_{0}+\mathfrak{B}^{-j+m} \subset \mathfrak{B}^{M-s} .
$$

Moreover for any $j_{1}<j_{2} \leq-M$, it can be easily verified that

$$
\left\{\left(\mathfrak{p}^{-1} N\right)^{-j_{1}} \xi_{0}+\mathfrak{B}^{-j_{1}+m}\right\} \cap\left\{\left(\mathfrak{p}^{-1} N\right)^{-j_{2}} \xi_{0}+\mathfrak{B}^{-j_{2}+m}\right\}=\Phi .
$$

Using (3.8) and (3.9), we have

$$
I_{2} \leq \int_{\mathfrak{B}^{M-s}}\left|\widehat{\Psi}^{(-j)}(x)\right|^{2} d x<\varepsilon
$$

from which the result follows.

DEFINITION 3.4. Let $\mathbb{K}$ be a local field of positive characteristic $p \geq 0$ and $\mathfrak{p}$ be a prime element of $\mathbb{K}$. A collection of closed subspaces $\left\{V_{j}: j \in \mathbb{Z}\right\}$ of $L^{2}\left(K, \mathbb{C}^{M}\right)$ is called a vector valued nonuniform nonstationary multiresolution analysis (VVNUNMRA) if the following conditions hold:

(a) $V_{j} \subset V_{j+1}$ for all $j \in \mathbb{Z}$;

(b) $\bigcup_{j \in \mathbb{Z}} V_{j}$ is dense in $L^{2}\left(K, \mathbb{C}^{M}\right)$;

(c) $\bigcap_{j \in \mathbb{Z}} V_{j}=\{0\}$;

(d) for any $j \in \mathbb{Z}$ there is a function $\Phi^{(j)} \in V_{j}$ such that the sequence $\left\{\Phi^{(j)}\left(\cdot+\left(\mathfrak{p}^{-1} N\right)^{-j} \lambda\right): \lambda \in \Lambda\right\}$ forms a Riesz basis (or orthonormal basis) for $V_{j}$.

The sequence $\left\{\Phi^{(j)}\right\}_{j \in \mathbb{Z}}$ is called a scaling sequence for the given VVNUNMRA. If we denote by $P_{j}$, the orthogonal projector on $V_{j}$, then condition (b) of the Definition 3.4 implies that $\lim _{j \rightarrow \infty} P_{j} f=f$ for any $f \in L^{2}\left(K, \mathbb{C}^{M}\right)$. It then follows from the condition (d) that for any $f \in V_{j}$, the function $f\left(x+\left(\mathfrak{p}^{-1} N\right)^{j} \lambda\right)$ also belong to $V_{j}$ for any $\lambda \in \Lambda$. Without loss of generality, we assume that $\left\{\Phi^{(j)}\left(x+\left(\mathfrak{p}^{-1} N\right)^{j} \lambda\right)\right\}_{\lambda \in \Lambda}$ constitutes an orthonormal basis in $V_{j}$. 
PROPOSITION 3.5. If $\left\{V_{j}\right\}_{j \in \mathbb{Z}}$ is a NUNMRA, then there exists a vector valued nonstationary orthonormal wavelet bases $\left\{\Psi_{j, \lambda}\right\}_{j \in \mathbb{Z}, \lambda \in \Lambda}$, such that for any $f \in L^{2}\left(K, \mathbb{C}^{M}\right)$,

$$
P_{j+1} f=P_{j} f=\sum_{\lambda \in \Lambda}\left\langle f, \Psi_{j, \lambda}\right\rangle \Psi_{j, \lambda}
$$

PROOF. Let $W_{j}$ be an orthogonal complement of $V_{j}$ in $V_{j+1}$. Then

$$
W_{j} \perp W_{j^{\prime}}, \quad \text { for } \quad j \neq j^{\prime}
$$

and for $j_{0}<j$,

$$
V_{j}=V_{j_{0}} \oplus\left(\bigoplus_{\ell=j_{0}}^{j-1} W_{\ell}\right) .
$$

It then follows from the conditions (b) and (d) of the definition 3.4 that

$$
L^{2}\left(K, \mathbb{C}^{M}\right)=\bigoplus_{j \in \mathbb{Z}} W_{j}
$$

Equation (3.10) is equivalent to the fact that for fixed $j$, the sequence $\left\{\Psi_{j, \lambda}\right\}_{\lambda \in \Lambda}$ forms an orthonormal basis in $W_{j}$. From (3.13), it follows that $\left\{\Psi_{j, \lambda}\right\}_{j \in \mathbb{Z}, \lambda \in \Lambda}$ is an orthonormal basis in $L^{2}\left(K, \mathbb{C}^{M}\right)$. Hence the problem of construction of nonstationary wavelet bases satisfying (3.10) is to find $\Psi^{(j)}$ such that $\left\{\Psi^{(j)}\left(x+\left(\mathfrak{p}^{-1} N\right)^{j} \lambda\right)\right\}_{\lambda \in \Lambda}$ constitutes an orthonormal basis in $W_{j}$.

For the construction of the function $\Psi^{(j)}$, we use the following properties of $\varphi^{(j)}$ and $W_{j}$.

Since $\Phi^{(j)} \subset V_{j} \subset V_{j+1}$ and $\left\{\Phi_{\lambda}^{(j+1)}\right\}_{\lambda \in \mathbb{N}_{0}}$ is an orthonormal basis in $V_{j+1}$, it follows that

$$
\Phi^{(j)}(x)=\sum_{\lambda \in \Lambda} h_{j+1, \lambda} \Phi_{\lambda}^{(j+1)}(x)
$$

where

$$
h_{j+1, \lambda}=\left\langle\Phi^{(j)}, \Phi^{(j+1)}\right\rangle, \quad \sum_{\lambda \in \Lambda}\left|h_{j+1, \lambda}\right|^{2}=1 .
$$

Equation (3.14) can be written in the frequency domain as

$$
\widehat{\Phi}^{(j)}(\xi)=m_{j+1}\left(\left(\mathfrak{p}^{-1} N\right)^{j+1} \xi\right) \widehat{\varphi}^{(j+1)}(\xi),
$$


where

$$
m_{j+1}(\xi)=\sum_{\lambda \in \mathbb{N}_{0}} h_{j+1, \lambda} \chi_{\lambda}(\xi),
$$

are called vector valued nonuniform nonstationary masks. It can be easily verified that

$$
\sum_{\lambda \in \Lambda}\left|\Phi^{(j)}\left(\xi+\left(\mathfrak{p}^{-1} N\right)^{-j} \lambda\right)\right|^{2}=(q N)^{-j} \quad \text { for a.e } \xi \in K .
$$

From (3.16) and (3.17), we have

$$
\sum_{\lambda \in \Lambda}\left|m_{j+1}\left(\left(\mathfrak{p}^{-1} N\right)^{j+1} \xi+\mathfrak{p} \lambda\right) \Phi^{(j+1)}\left(\xi+\left(\mathfrak{p}^{-1} N\right)^{j} \lambda\right)\right|^{2}=(q N)^{-j} .
$$

Partitioning the sum into two parts and taking into account the integral periodicity of $m_{j+1}$, we get

$$
\left|m_{j+1}(\xi)\right|^{2}+\left|m_{j+1}(\xi+\mathfrak{p} u(N))\right|^{2}=q N .
$$

We now characterize the subspaces $W_{j}$. Let $f \in W_{j}$. Then $f$ is in $V_{j+1}$ and is orthogonal to $V_{j}$. Then

$$
f(x)=\sum_{k \in \mathbb{N}_{0}} f_{k} \Phi_{k}^{(j+1)}(x),
$$

where $f_{\lambda}=\left\langle f, \Phi_{\lambda}^{(j+1)}\right\rangle$. Applying Fourier transform to equation (3.19), we have

$$
\widehat{f}(\xi)=m_{f}\left(\left(\mathfrak{p}^{-1} N\right)^{j+1} \xi\right) \widehat{\Phi}^{(j+1)}(\xi),
$$

where

$$
m_{f}(\xi)=\sum_{\lambda \in \Lambda} f_{\lambda} \chi_{\lambda}(\xi),
$$

are integral periodic from $L^{2}(\mathfrak{D})$. Since $f$ is orthogonal to $V_{j}$, we have for $\lambda \in \Lambda$,

$$
\int_{\mathbb{K}} \widehat{f}(\xi) \overline{\widehat{\Phi}^{(j)}(\xi)} \chi_{\lambda}\left(\left(\mathfrak{p}^{-1} N\right)^{j-1} \xi\right) d \xi=0 .
$$


Moreover,

$$
\begin{aligned}
\int_{\mathbb{K}} \widehat{f}(\xi) & \overline{\widehat{\Phi}^{(j)}(\xi)} \chi_{\lambda}\left(\left(\mathfrak{p}^{-1} N\right)^{j-1} \xi\right) d \xi \\
& =\int_{\left(\mathfrak{p}^{-1} N\right)^{j} \mathfrak{D}} \sum_{r \in \mathbb{N}_{0}} \widehat{f}\left(\xi+\left(\mathfrak{p}^{-1} N\right)^{-j} u(r)\right) \overline{\widehat{\Phi}^{(j)}\left(\xi+\left(\mathfrak{p}^{-1} N\right)^{-j} u(r)\right)} \chi_{\lambda}\left(\left(\mathfrak{p}^{-1} N\right)^{j-1} \xi\right) d \xi \\
& =0 .
\end{aligned}
$$

Since (3.21) holds for all $\lambda \in \Lambda$, we have

$$
\sum_{r \in \mathbb{N}_{0}} \widehat{f}\left(\xi+\left(\mathfrak{p}^{-1} N\right)^{-j} u(r)\right) \overline{\widehat{\Phi}^{(j)}\left(\xi+\left(\mathfrak{p}^{-1} N\right)^{j} u(r)\right)}=0 .
$$

The series in (3.22) converges in $L^{2}(\mathfrak{D})$. Keeping in view (3.18) and using equations (3.20) and (3.21) in (3.22), we get

$$
\begin{aligned}
(q N)^{j+1} & \sum_{r \in \mathbb{N}_{0}} \widehat{f}\left(\xi+\left(\mathfrak{p}^{-1} N\right)^{-j} u(r)\right) \overline{\widehat{\Phi}^{(j)}\left(\xi+\left(\mathfrak{p}^{-1} N\right)^{-j} u(r)\right)} \\
\quad= & m_{f}\left(\left(\mathfrak{p}^{-1} N\right)^{j+1} \xi\right) \overline{m_{j+1}\left(\left(\mathfrak{p}^{-1} N\right)^{j+1} \xi\right)} m_{f}\left(\left(\mathfrak{p}^{-1} N\right)^{j+1} \xi+\mathfrak{p} u(N)\right) \overline{m_{j+1}\left(\left(\mathfrak{p}^{-1} N\right)^{j+1} \xi+\mathfrak{p} u(N)\right)} \\
\quad & 0 .
\end{aligned}
$$

It is evident from (3.18) that $\overline{m_{j+1}(\xi)}$ and $\overline{m_{j+1}(\xi+\mathfrak{p} u(N))}$ can not vanish simultaneously. Hence, there exist integral periodic function $\lambda(\xi)$ such that

$$
m_{f}(\xi)=\lambda(\xi) \overline{m_{j+1}(\xi+\mathfrak{p} u(N))} \text { a.e. }
$$

and

$$
\lambda(\xi)+\lambda(\xi+\mathfrak{p} u(N))=0
$$

Equation (3.24) can be rewritten as

$$
\lambda(\xi)=v\left(\mathfrak{p}^{-1} N \xi\right) \overline{\chi(\xi)}
$$

where $v$ is an integral periodic function. Therefore the Fourier transform of any function of $W_{j}$ yields

$$
\widehat{f}(\xi)=\overline{m_{j+1}(\xi+\mathfrak{p} u(N))} v\left(\left(\mathfrak{p}^{-1} N\right)^{j} \xi\right) \widehat{\Phi}^{(j+1)}(\xi) \overline{\chi\left(\mathfrak{p}^{j} \xi\right)} .
$$

Moreover, it can be seen that $v$ is square integrable. Having system (3.25) in hand, it will not be difficult to find functions $\Psi^{(j)}$ in the $W_{j}$ space such that 
$\left\{\Psi^{(j)}\left(x+\left(\mathfrak{p}^{-1} N\right)^{-j} \lambda\right)\right\}_{\lambda \in \Lambda}$ constitutes an orthonormal basis in $W_{j}$. Therefore, we have

$$
\widehat{\Psi}^{(j)}(\xi)=\overline{m_{j+1}(\xi+\mathfrak{p} u(N))} v_{\Psi(j)}\left(\left(\mathfrak{p}^{-1} N\right)^{j} \xi\right) \widehat{\Phi}^{(j+1)}(\xi) \overline{\chi\left(\left(\mathfrak{p}^{-1} N\right)^{j} \xi\right)} .
$$

Therefore, substituting above expression in (3.17) and using (3.18), we have

$$
\left|v_{\Psi(j)}\right|^{2}=1 \quad \text { a.e. }
$$

From (3.25), it follows that the integer shifts of $\Psi^{(j)}$ defined by

$$
\widehat{\Psi}^{(j)}(\xi)=\overline{m_{j+1}(\xi+\mathfrak{p} u(N))} \widehat{\Phi}^{(j+1)}(\xi) \overline{\chi\left(\left(\mathfrak{p}^{-1} N\right)^{j} \xi\right)} .
$$

forms a basis of $W_{j}$. Thus having a nonstationary multiresolution analysis $\left\{V_{j}\right\}_{j \in \mathbb{Z}}$ generated by a scaling function $\left\{\Phi^{(j)}\right\}$, one can construct a nonstationary orthonormal wavelet basis $\left\{\Psi_{j, \lambda}\right\}_{j \in \mathbb{Z}, \lambda \in \Lambda}$ in $L^{2}\left(K, \mathbb{C}^{M}\right)$ satisfying (3.10).

DEFINITION 3.6. Suppose $\Psi^{(j)} \in L^{2}\left(K, \mathbb{C}^{M}\right)$ for $j \in \mathbb{Z}$. Then dimension function is defined by

$$
D_{\Psi(j)}(\xi)=\sum_{n=1}^{\infty} \sum_{\lambda \in \Lambda}\left|\widehat{\Psi}^{(j-n)}\left(\left(\mathfrak{p}^{-1} N\right)^{-n}(\xi+\lambda)\right)\right|^{2} \quad \text { a.e } \xi \in K
$$

Since

$$
\int_{\mathfrak{D}} \sum_{n=1}^{\infty} \sum_{\lambda \in \Lambda}\left|\widehat{\Psi}^{(j-n)}\left(\left(\mathfrak{p}^{-1} N\right)^{-n}(\xi+\lambda)\right)\right|^{2} d \xi=\sum_{n=1}^{\infty}(q N)^{-n} \int_{\mathbb{K}}\left|\widehat{\psi}^{(j)}(\xi)\right|^{2} d \xi .
$$

Hence $D_{\Psi^{(j)}}$ is well defined for a.e. $\xi \in K$.

PROPOSITION 3.7. For all $j \in \mathbb{Z}$ and for a.e. $\xi \in K$, we have

$$
\left|\widehat{\Phi}^{(j)}\right|^{2}=\sum_{n=1}^{\infty}\left|\widehat{\Psi}^{(j-n)}\left(\left(\mathfrak{p}^{-1} N\right)^{-n} \xi\right)\right|^{2} .
$$


PROOF. If $\Psi^{(j)} \in L^{2}\left(K, \mathbb{C}^{M}\right)$, then equation (3.26) holds. Therefore using (3.18), we have from equations (3.16) and (3.26),

$$
\begin{aligned}
\left|\widehat{\Phi}^{(j)}(\xi)\right|^{2}+\left|\widehat{\Psi}^{(j)}(\xi)\right|^{2} & =\left|m_{j+1}\left(\left(\mathfrak{p}^{-1} N\right)^{j+1} \xi\right) \widehat{\Phi}^{(j+1)}(\xi)\right|^{2} \\
& +\left|\overline{m_{j+1}(\xi+\mathfrak{p} u(N))} \widehat{\Phi}^{(j+1)}(\xi) \overline{\chi\left(\left(\mathfrak{p}^{-1} N\right)^{-j} \xi\right)}\right|^{2} \\
& =q N\left|\widehat{\Phi}^{(j+1)}(\xi)\right|^{2} \\
& =\left|\widehat{\Phi}^{(j+1)}\left(\mathfrak{p}^{-1} N \xi\right)\right|^{2} .
\end{aligned}
$$

Since the equality holds for a.e. $\xi \in K$, we have

$$
\left|\widehat{\Phi}^{(j)}(\xi)\right|^{2}=\left|\widehat{\Phi}^{(j-1)}\left(\mathfrak{p}^{-1} N \xi\right)\right|^{2}+\left|\widehat{\Psi}^{(j-1)}\left(\mathfrak{p}^{-1} N \xi\right)\right|^{2} .
$$

Iterating for any integer $L \geq 1$, we get

$$
\left|\widehat{\Phi}^{(j)}(\xi)\right|^{2}=\left|\widehat{\Phi}^{(j-L)}\left(\left(\mathfrak{p}^{-1} N\right)^{-L} \xi\right)\right|^{2}+\sum_{n=1}^{L}\left|\widehat{\Psi}^{(j-L)}\left(\left(\mathfrak{p}^{-1} N\right)^{-n} \xi\right)\right|^{2} .
$$

Since $\left|\widehat{\Phi}^{(j-L)}(\xi)\right| \leq 1$, the sequence

$$
\left\{\sum_{n=1}^{L}\left|\widehat{\Psi}^{(j-L)}\left(\left(\mathfrak{p}^{-1} N\right)^{-n} \xi\right)\right|^{2}: L \geq 1\right\}
$$

of real numbers is bounded by 1 , hence it converges. Therefore, $\lim _{L \rightarrow \infty}\left|\widehat{\Phi}^{(j-L)}\left(\left(\mathfrak{p}^{-1} N\right)^{-L} \xi\right)\right|^{2}$ also exists. Moreover

$$
\int_{\mathbb{K}}\left|\widehat{\Phi}^{(j-L)}\left(\left(\mathfrak{p}^{-1} N\right)^{-L} \xi\right)\right|^{2} d \xi=(q N)^{-L} \int_{\mathbb{K}}\left|\widehat{\Phi}^{(j-L)}(\xi)\right|^{2} d \xi \rightarrow 0 \quad \text { as } L \rightarrow \infty .
$$

Therefore, the application of the Fatou's Lemma yields

$$
\int_{\mathbb{K}} \lim _{L \rightarrow \infty}\left|\widehat{\Phi}^{(j-L)}\left(\left(\mathfrak{p}^{-1} N\right)^{-L} \xi\right)\right|^{2} d \xi \leq \lim _{L \rightarrow \infty} \int_{\mathbb{K}}\left|\widehat{\Phi}^{(j-L)}\left(\left(\mathfrak{p}^{-1} N\right)^{-L} \xi\right)\right|^{2} d \xi=0 .
$$

This means that $\lim _{L \rightarrow \infty}\left|\widehat{\Phi}^{(j-L)}\left(\left(\mathfrak{p}^{-1} N\right)^{-L} \xi\right)\right|^{2} d \xi=0$. Hence, we have

$$
\left|\widehat{\Phi}^{(j)}(\xi)\right|^{2}=\sum_{n=1}^{\infty}\left|\widehat{\Psi}^{(j-n)}\left(\left(\mathfrak{p}^{-1} N\right)^{-n} \xi\right)\right|^{2}
$$

This completes the proof. 
Since $\left\{(q N)^{j / 2} \Phi^{(j)}\left(\left(\mathfrak{p}^{-1} N\right)^{j} x-\lambda\right): \lambda \in \Lambda\right\}$ is an orthonormal basis of $L^{2}\left(K, \mathbb{C}^{M}\right)$ for all $j \in \mathbb{Z}$, we have

$$
1=\sum_{\lambda \in \Lambda}\left|\widehat{\Phi}^{(j)}(\xi+\lambda)\right|^{2}=\sum_{\lambda \in \Lambda} \sum_{n=1}^{\infty}\left|\widehat{\Psi}^{(j-n)}\left(\left(\mathfrak{p}^{-1} N\right)^{-n}(\xi+\lambda)\right)\right|^{2}=D_{\Psi^{(j)}}(\xi) .
$$

Since $D_{\Psi^{(j)}}(\xi)=1$, we can choose the smallest $n \in \mathbb{N}$ such that for all $j \in \mathbb{Z}$ and for almost all $\xi \in K$,

$$
\sum_{\lambda \in \Lambda}\left|\widehat{\Psi}^{(j-n)}\left(\left(\mathfrak{p}^{-1} N\right)^{-n}(\xi+\lambda)\right)\right|^{2} \neq 0
$$

and then for almost all $\xi \in K$, we define $\Phi^{(j)}(\xi)$ by

$$
\widehat{\Phi}^{(j)}(\xi)=\frac{\widehat{\Psi}^{(j-n)}\left(\left(\mathfrak{p}^{-1} N\right)^{-n} \xi\right)}{\sqrt{\sum_{\lambda \in \Lambda}\left|\widehat{\Psi}^{(j-n)}\left(\left(\mathfrak{p}^{-1} N\right)^{-n}(\xi+\lambda)\right)\right|^{2}}} .
$$

Moreover for a fixed $j \in \mathbb{Z}$ and $n \in \mathbb{N}$, we define an infinite vector of $l^{2}(\Lambda)$ as

$$
\left.\Psi_{j, n}(\xi)=\left\{\widehat{\Psi}^{(j-n)}\left(\left(\mathfrak{p}^{-1} N\right)^{-n}(\xi+\lambda)\right)\right)\right\}_{\lambda \in \lambda} \quad \text { for a.e } \xi \in K
$$

THEOREM 3.8. Assume that $\Psi^{(j)} \in L^{2}\left(K, \mathbb{C}^{M}\right)$ for every $j \in \mathbb{Z}$, such that the system $\left\{(q N)^{j / 2} \Psi^{(j)}\left(\left(\mathfrak{p}^{-1} N\right)^{j} x-\lambda\right): \lambda \in \Lambda\right\}$ is an orthonormal basis of $L^{2}\left(K, \mathbb{C}^{M}\right)$. Then the mother wavelets $\Psi^{(j)}, j \in \mathbb{Z}$ come from a VVNUNMRA, if and only if

$$
D_{\Psi(j)}(\xi)=\sum_{n=1}^{\infty} \sum_{k \in \mathbb{N}_{0}}\left|\widehat{\Psi}^{(j-n)}\left(\left(\mathfrak{p}^{-1} N\right)^{-n}(\xi+\lambda)\right)\right|^{2}=1 \quad \text { a.e } \xi \in K
$$

PROOF. Necessary part of the Theorem follows from the Proposition 3.7. For the proof of the sufficient part, we need the following lemmas:

LEMMA 3.9. For all $j \in \mathbb{Z}$, and for almost all $\xi \in K$, we have

$$
\Psi_{j, n}(\xi)=\sum_{h=1}^{\infty}\left\langle\Psi_{j, n}(\xi), \Psi_{j, h}(\xi)\right\rangle \Psi_{j, h}(\xi)
$$


PROOF. The series in the Lemma converges absolutely for a.e. $\xi \in K$. Let us first show that

$$
\begin{aligned}
& \widehat{\Psi}^{(j-n)}\left(\left(\mathfrak{p}^{-1} N\right)^{-n} \xi\right)=\sum_{h=1}^{\infty} \sum_{\lambda \in \Lambda} \widehat{\Psi}^{(j-n)}\left(\left(\mathfrak{p}^{-1} N\right)^{-n}(\xi+\lambda)\right)
\end{aligned}
$$

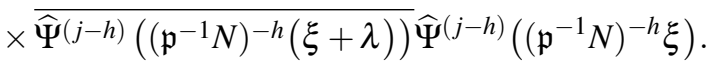

Let us denote by $\Gamma_{j, n}(\xi)$, the second member of the series (3.30). Then using Equation (3.7) and (3.30), we have

$$
\begin{aligned}
& \Gamma_{j, n}(\xi)=\sum_{h=1}^{\infty} \sum_{\lambda \in \Lambda} \widehat{\Psi}^{(j-n)}\left(\left(\mathfrak{p}^{-1} N\right)^{-n}(\xi+\lambda)\right) \overline{\widehat{\Psi}^{(j-h)}\left(\left(\mathfrak{p}^{-1} N\right)^{-h}(\xi+\lambda)\right)} \widehat{\Psi}^{(j-h)}\left(\left(\mathfrak{p}^{-1} N\right)^{-h} \xi\right) \\
& =\sum_{\lambda \in \Lambda} \widehat{\Psi}^{(j-n)}\left(\left(\mathfrak{p}^{-1} N\right)^{-n}(\xi+\lambda)\right)\left\{\sum_{h=0}^{\infty} \overline{\widehat{\Psi}^{(j-h)}\left(\left(\mathfrak{p}^{-1} N\right)^{-h}(\xi+\lambda)\right)} \widehat{\Psi}^{(j-h)}\left(\left(\mathfrak{p}^{-1} N\right)^{-h} \xi\right)\right. \\
& \left.-\widehat{\widehat{\Psi}^{(j)}(\xi+\lambda)} \widehat{\Psi}^{(j)}(\xi)\right\} \\
& =\sum_{h=0}^{\infty} \sum_{\lambda q \in \Lambda} \widehat{\Psi}^{(j-n)}\left(\left(\mathfrak{p}^{-1} N\right)^{-n}(\xi+u(q \lambda))\right) \\
& \times \overline{\widehat{\Psi}^{(j-h)}\left(\left(\mathfrak{p}^{-1} N\right)^{-h}(\xi+u(q k))\right)} \widehat{\Psi}^{(j-h)}\left(\left(\mathfrak{p}^{-1} N\right)^{-h} \xi\right) \\
& =\sum_{h=1}^{\infty} \sum_{\lambda \in \Lambda} \widehat{\Psi}((j+1)-(n+1))\left(\left(\mathfrak{p}^{-1} N\right)^{n+1}(\mathfrak{p} \xi+\lambda)\right) \\
& \times \overline{\widehat{\Psi}^{(j-h)}\left(\left(\mathfrak{p}^{-1} N\right)^{-h}(\mathfrak{p} \xi+\lambda)\right)} \widehat{\Psi}^{(j-h)}\left(\left(\mathfrak{p}^{-1} N\right)^{-h} \mathfrak{p} \xi\right) \\
& =\Gamma_{j+1, n+1}(\mathfrak{p} \xi)
\end{aligned}
$$

The above system is equivalent to

$$
\Gamma_{j, n}(\xi)=\Gamma_{j-1, n-1}\left(\mathfrak{p}^{-1} N \xi\right) .
$$

In consequence, for $j \in \mathbb{Z}, n \in \mathbb{N}$ and almost all $\xi \in \mathbb{K}$, we have by recursion

$$
\Gamma_{j, n}(\xi)=\Gamma_{j-(n-1), 1}\left(\left(\mathfrak{p}^{-1} N\right)^{n+1} \xi\right)
$$

from which equation (3.30) follows as $\Gamma_{j-n+1,1}(\xi)=\widehat{\Psi}^{(j-n)}\left(\left(\mathfrak{p}^{-1} N\right)^{-n} \xi\right)$.

Moreover, since $\left\langle\Psi_{j, n}(\xi), \Psi_{j, h}(\xi)\right\rangle$ is integral periodic, equation (3.29) holds. This completes the proof of the lemma. 
From the above lemma, it can be seen that

$$
\sum_{n=1}^{\infty}\left\|\Psi_{j, n}(\xi)\right\|_{l^{2}(\Lambda)}^{2}=\sum_{n=1}^{\infty} \sum_{\lambda \in \Lambda}\left|\widehat{\Psi}^{(j-n)}\left(\left(\mathfrak{p}^{-1} N\right)^{-n}(\xi+\lambda)\right)\right|^{2}=D_{\Psi^{(j)}}(\xi)=1 .
$$

For all $j \in \mathbb{Z}$, and for almost all $\xi \in K$, we define

$$
\mathscr{F}_{j}(\xi)=\overline{\operatorname{span}}\left\{\Psi_{j, n}(\xi): n \geq 1\right\} .
$$

It is a subspace of $l^{2}(\Lambda)$ of dimension 1 .

LEMMA 3.10. Let $\left\{\alpha_{n}: n \geq 1\right\}$ be a family of vectors in a Hilbert space $\mathbb{H}$ such that

$$
\sum_{n=1}^{\infty}\left\|\alpha_{n}\right\|^{2}=C<\infty \quad \text { and } \quad v_{n}=\sum_{m=1}^{\infty}\left\langle\alpha_{n}, \alpha_{m}\right\rangle \alpha_{m} \quad \text { for all } n \geq 1 .
$$

Then dimension of the subspace $\overline{\operatorname{span}}\left\{\alpha_{n}: n \geq 1\right\}$ of $\mathbb{H}$ is equal to $C$.

Sufficient part of the Theorem: Using Lemma 3.10, it follows that the family $\mathscr{F}_{j}(\xi)$, defined by (3.32) is generated by only one unit vector $X_{j}(\xi)$. To construct it, we first make a partition of $\mathfrak{D}$ as follows

$$
\mathscr{P}_{j, n}=\left\{\xi \in \mathfrak{D}: \Psi_{j, n}(\xi) \neq 0 \quad \text { and } \quad \Psi_{j, m}(\xi)=0 \quad \text { for } m<n\right\}, n \geq 1,
$$

and the null set

$$
\mathscr{P}_{j, 0}=\left\{\xi \in \mathfrak{D}: D_{\Psi(j)}(\xi)=0\right\} .
$$

Let us now define the unit vector $X_{j}(\xi)$ on $\mathfrak{D}$ by

$$
X_{j}(\xi)=\frac{\Psi_{j, n}(\xi)}{\left\|\Psi_{j, n}(\xi)\right\|_{l^{2}(\Lambda)}} \quad \text { if } \xi \in E_{j, n}
$$

We write $X_{j}(\xi)=\left\{u_{\lambda}^{(j)}(\xi)\right\}_{\lambda \in \Lambda}$ and define $\Phi^{(j)}$ almost everywhere on $\mathbb{K}$ by

$$
\widehat{\Phi}^{(j)}(\xi)=u_{\lambda}^{(j)}(\xi-\lambda) \quad \text { if } \xi \in \mathfrak{D}+\lambda .
$$

These $\Phi^{(j)}, j \in \mathbb{Z}$ are the required scaling functions. 


\section{REFERENCES}

O. Ahmad, M.Y. Bhat, N. A. Sheikh, Construction of Parseval Framelets Associated with GMRA on Local Fields of Positive Characteristic, Numerical Functional Analysis and optimization (2021), https://doi.org/10.1080/01630563.2021.1878370.

O. Ahmad, N. Ahmad, Construction of Nonuniform Wavelet Frames on Non-Archimedean Fields, Math. Phy. Anal. and Geometry, 23 (47) (2020).

O. Ahmad, N. A Sheikh, K. S Nisar, F. A. Shah, Biorthogonal Wavelets on Spectrum, Math. Methods in Appl. Sci, (2021) 1-12. https://doi.org/10.1002/mma.7046.

O. Ahmad, Nonuniform Periodic Wavelet Frames on Non-Archimedean Fields, Annales Universitatis Mariae Curie-Sklodowska, sectio A - Mathematica, 1-17, (2) (2020) doi: 10.17951/a.2020.74.2.1-17.

O. Ahmad, N. A Sheikh, Explicit Construction of Tight Nonuniform Framelet Packets on Local Fields, Operators and Matrices 15 (1) (2021), 131-149.

O. Ahmad, N.A. Sheikh, M. A. Ali, Nonuniform nonhomogeneous dual wavelet frames in Sobolev spaces in $L^{2}(\mathbb{K})$, Afr. Mat., (2020) doi.org/10.1007/s13370-020-00786-1.

O. Ahmad and N. A. Sheikh, On Characterization of nonuniform tight wavelet frames on local fields, Anal. Theory Appl., 34 (2018) 135-146.

O. Ahmad, F. A. Shah and N. A. Sheikh, Gabor frames on non-Archimedean fields, International Journal of Geometric Methods in Modern Physics, 15 (2018) 1850079 (17 pages).

S. Albeverio, S. Evdokimov, and M. Skopina, p-adic nonorthogonal wavelet bases, Proc. Steklov Inst. Math., 265 (2009), 135-146.

S. Albeverio, S. Evdokimov, and M. Skopina, $p$-adic multiresolution analysis and wavelet frames, J. Fourier Anal. Appl., 16 (2010), 693-714.

S. Albeverio, A. Khrennikov, and V. Shelkovich, Theory of p-adic Distributions: Linear and Nonlinear Models, Cambridge University Press, 2010.

S. Albeverio, R. Cianci, and A. Yu. Khrennikov, p-Adic valued quantization, p-Adic Numbers Ultrametric Anal. Appl. 1, 91-104 (2009).

J. J. Benedetto and R. L. Benedetto, A wavelet theory for local fields and related groups, J. Geom. Anal. 14 (2004) 423-456.

F. Bastin and L. Simons, About Nonstationary Multiresolution Analysis and Wavelets, Results. Math. 63 (2013), 485-500.

M. Z. Berkolayko, I. Y. Novikov, On infinitely smooth compactly supported almost-wavelets, Math. Notes 56 (3-4) (1994) 877-883.

C. de Boor, R. DeVore, A. Ron, On the construction of multivariate (pre)wavelets, Constr. Approx. 9 (1993) 123-166.

I. Daubechies, Ten Lectures on Wavelets, CBMS-NSF Series in Applied Mathematics, SIAM, Philadelphia, 1992.

S. Evdokimov and M. Skopina, 2-adic wavelet bases, Proc. Steklov Inst. Math., 266 (2009), S143-S154

E. A. Lebedeva, On a connection between nonstationary and periodic wavelets, J. Math. Anal. Appl.

Y. Farkov, Orthogonal wavelets on locally compact abelian groups, Funct. Anal. Appl., 31 (1997), 451 (1) (2017) 434-447. 
Y. Farkov, Multiresolution Analysis and Wavelets on Vilenkin Groups, Facta Universitatis (NIS), Ser.: Elec. Energ., 21 (2008), 309-325.

J. P. Gabardo and M. Nashed, Nonuniform multiresolution analyses and spectral pairs, J. Funct. Anal. 158 (1998) 209-241.

J. P. Gabardo and X. Yu, Wavelets associated with nonuniform multiresolution analyses and onedimensional spectral pairs, J. Math. Anal. Appl., 323 (2006) 798-817.

H. K. Jiang, D.F. Li and N. Jin, Multiresolution analysis on local fields, J. Math. Anal. Appl. 294 (2004) 523-532.

A.Khrennikov andV.Shelkovich, Non-Haar $p$-adic wavelets and their application to pseudo-differential operators and equations, Appl. Comput. Harmon. Anal., 28 (2010) 1-23.

A. Khrennikov, V. Shelkovich, and M. Skopina, $p$-adic refinable functions and MRA-based wavelets, $J$. Approx. Theory. 161 (2009), 226-238.

A. Khrennikov, K. Oleschko, M.J.C. López, Application of p-adic wavelets to model reaction-diffusion dynamics in random porous media. J. Fourier Anal. Appl., 22 (2016) 809-822.

A. Khrennikov, Modeling of Processes of Thinking in p-adic Coordinates [in Russian], Fizmatlit, Moscow (2004).

S. Kozyrev and A. Khrennikov, p-adic integral operators in wavelet bases, Doklady Math., 83 (2011), 209-212.

S. Kozyrev, A. Khrennikov, and V. Shelkovich, p-Adic wavelets and their applications, Proc. Steklov Inst. Math., 285 (2014), 157-196.

W. C. Lang, Orthogonal wavelets on the Cantor dyadic group, SIAM J. Math. Anal., 27 (1996), 305-312.

W. C. Lang, Wavelet analysis on the Cantor dyadic group, Houston J. Math., 24 (1998), 533-544.

W. C. Lang, Fractal multiwavelets related to the cantor dyadic group, Int. J. Math. Math. Sci. 21 (1998), 307-314.

D. F. Li and H. K. Jiang, The necessary condition and sufficient conditions for wavelet frame on local fields, J. Math. Anal. Appl. 345 (2008) 500-510.

S. G. Mallat, Multiresolution approximations and wavelet orthonormal bases of $L^{2}(\mathbb{R})$, Trans. Amer. Math. Soc. 315 (1989) 69-87.

S. G. Mallat, A Wavelet Tour of Signal Processing, Academic Press Inc., 1st Edition 1998, 2nd Edition 1999.

F.A. Shah and O. Ahmad, Wave packet systems on local fields , Journal of Geometry and Physics, 120 (2017) 5-18.

F. A. Shah, O. Ahmad and A. Rahimi, Frames Associated with Shift Invariant Spaces on Local Fields, Filomat 32 (9) (2018) 3097-3110.

F. A. Shah and Abdullah, Nonuniform multiresolution analysis on local fields of positive characteristic, Complex Anal. Opert. Theory, 9 (2015) 1589-1608.

M. H. Taibleson, Fourier Analysis on Local Fields, Princeton University Press, Princeton, NJ, 1975. 
Owais Ahmad

Department of Mathematics,

National Institute of Technology,

Hazratbal, Srinagar -190 006, Jammu and Kashmir, India.

E-mail: siawoahmad@gmail.com; neyaznit@yahoo.co.in

Abid H. Wani

Department of Computer Science,

University of Kashmir,

South Campus, Anantnag -192101, Jammu and Kashmir, India.

E-mail: abid.wani@uok.edu.in

Neyaz A. Sheikh

Department of Mathematics,

National Institute of Technology,

Hazratbal, Srinagar -190 006, Jammu and Kashmir, India.

E-mail:neyaznit@yahoo.co.in

\section{Mobin Ahmad}

Department of Mathematics,

Faculty of Science, Jazan University,

Jazan -45142, Saudi Arabia.

E-mail: msyed@jazanu.edu.sa 